\title{
SQUARING OPERATIONS IN THE ADAMS SPECTRAL SEQUENCE
}

\author{
BY DANIEL S. KAHN
}

Communicated by P. Emery Thomas, August 26, 1968

It has been long known that the cohomology $H(A)$ of the mod 2 Steenrod algebra $A$ admits squaring operations. (For example, see [6].) Since $H(A)$ is isomorphic to the $E_{2}$ term of the mod 2 Adams spectral sequence [2], it is natural to inquire as to the relation of these squaring operations to the structure of the Adams spectral sequence. In this note we announce some results of this type extending those of [5]. Details will appear elsewhere.

The author is indebted to J. F. Adams, M. G. Barratt and M. Mahowald for conversations and correspondence which were helpful in the present work.

1. The quadratic construction. The main tool in this study is the quadratic construction, a functor from pointed spaces to filtered spaces which has been studied by J. F. Adams, M. G. Barratt and M. Mahowald (unpublished).

Let $X$ be a finite CW complex with basepoint. Denote by $Q^{\prime n}(X)$ the space $S^{n} \ltimes(X \wedge X)$. (If $B$ is a space with basepoint $b, A \ltimes B$ shall mean $A \quad B / A \times b$.) Define the involution $T: Q^{\prime n}(X) \rightarrow Q^{\prime n}(X)$ by $T(x, y, z)=(-x, z, y)$, where $-x$ is the point antipodal to $x$. This defines an action of $Z_{2}$ on $Q^{\prime n}(X)$ and we set $Q^{n}(X)=Q^{\prime n}(X) / Z_{2}$. $Q(X)=Q^{\infty}(X)$ is called the quadratic construction on $X$ and is clearly a functor. It is not difficult to show that $Q^{r}\left(S^{n}\right)$ is homeomorphic to $S^{n} \wedge P_{n}^{n+r}$, where $P_{n}^{n+r}$ denotes the stunted real projective space $R P^{n+r} / R P^{n-1}$.

Lemma (1.1). If $n=2^{q}$ where $q \geqq \phi(r)$ [3], $S^{n}=P_{n}^{n}$ is a retract of $P_{n}^{n+r}$.

Proof. [3], [4].

Denote by $\phi_{n, r}$ such a retraction.

Let $\bar{\alpha} \in \pi_{t}^{s}\left(S^{0}\right)=G_{t}$ correspond to $\alpha \in E_{2}^{s, s+t}\left(S^{0}\right)$ and let $\bar{\alpha}$ be represented by a map $f: S^{n+t} \rightarrow S^{n}$ where $n=2^{q}$ and $q \geqq \phi(r)$ (and perhaps much larger). Define $\Phi_{r}(f): S^{n+t} \wedge P_{n+t}^{n+t+r} \rightarrow S^{2 n}$ by $\Phi_{r}(f)=\left(S^{n} \wedge \phi_{n, r}\right)$ $\circ Q^{r}(f)$. Let

$$
\theta_{k}:\left(E^{2(n+t)+k}, S^{2(n+t)+k-1}\right) \rightarrow\left(S^{n+t} \wedge P_{n+t}^{n+t+k}, S^{n+t} \wedge P_{n+t}^{n+t+k-1}\right)
$$


denote the characteristic map of the cell of dimension $2(n+t)+k$ in $Q^{r}\left(S^{n+t}\right)$. Finally, let $W_{0} \supset W_{1} \supset W_{2} \supset \ldots$ be a realization [2] of $B(A)$, the bar construction on $A[1]$ with $W_{0}$ having the homotopy type of $S^{2 n}$. With this notation and hypotheses, we state

ThEOREM (1.2). $\Phi_{r}(f)$ is homotopic to a map $\Theta: S^{n+t} \wedge P_{n+t}^{n+t+r} \rightarrow W_{0}$ such that

(A) $\Theta\left(S^{n+t} \wedge P_{n+t}^{n+t+k}\right) \subset W_{2 s-k}, 0 \leqq k \leqq r$, and

(B) the composite $\Theta \circ \theta_{k}$ represents a cycle of $E_{1}^{2 s-1,2 s+2 t}\left(S^{0}\right)$ whose image in $E_{2}^{2 s-k, 2 s+2 t}\left(S^{0}\right)$ is $\alpha \cup_{k} \alpha$.

This theorem proves an early conjecture in the study of the quadratic construction.

2. Some differentials. Knowledge of the cell structure of $P_{m}^{m+r}$ together with Theorem (1.2) yields information concerning certain differentials in the Adams spectral sequence.

If $m=(2 a+1) 2^{c+4 d}, 0 \leqq c \leqq 3$, then $\rho(m)=2^{c}+8 d$. With this notation we state

TheOREM (2.1). Let $\alpha \in E_{2}^{s, s+t}\left(S^{0}\right)$ be a permanent cycle. If $k+1$ $\leqq \rho(t+k+1), \alpha \cup_{k} \alpha$ is also a permanent cycle.

(2.1) follows from (1.2) and the fact that $P_{2^{2}+t}^{2^{q}+t+k}$ is reducible [4, p. 214] if $k+1 \leqq \rho\left(2^{q}+t+k+1\right)=\rho(t+k+1)$ if $q$ is large enough [3], [4].

Similar arguments can be used to prove the following theorem.

Theorem (2.2). Let $i=0,1,2$ or 3 . If $\alpha \in E_{2}^{s, s+t}\left(S^{0}\right)$ is a permanent cycle and that

(i) $k+1 \leqq \rho(t+k+1)$, and

(ii) $t+k \equiv 2^{i+1}-1 \bmod 2^{i+1}$,

then $\alpha \cup_{k+2^{i}} \alpha$ survives to $E_{2^{i}+1}\left(S^{0}\right)$ and

$$
\delta_{2^{i}+1}\left(\alpha \cup_{k+2^{i}} \alpha\right)=h_{i}\left(\alpha \cup_{k} \alpha\right) .
$$

For $k=0$, Theorem (2.2) corresponds to identities in $G *$ such as $2 \bar{\alpha}^{2}=0$ if $\bar{\alpha} \in G_{2 r+1}$ or $\eta \bar{\alpha}^{2}=0$ if $\alpha \in G_{4 r+3}$. These identities are provable using the quadratic construction.

3. Homotopy operations. The quadratic construction has been used by Adams, Barratt, B. Gray and Mahowald to define cup- $i$ products in $G_{*}$. We discuss two examples of such operations and their relation to the cup- $i$ products in the Adams spectral sequence.

Let $\bar{\alpha} \in G_{t}$ be represented by $f: S^{n+t} \rightarrow S^{n}, n=2^{q}, q$ large. Let $\theta_{k}$ be as in $\$ 1$. Then the quadratic construction yields a null-homotopy $H$ of $\Phi_{r-1}(f) \circ \theta_{r-1}^{\prime}$, where $\theta_{r-1}^{\prime}=\theta_{r} \mid S^{2(n+t)+r-1}$. 
EXAMPLE A. If $r+1 \leqq \rho(t+r+1)$, then $\theta_{r-1}^{\prime}$ itself is null homotopic in $Q^{r-1}\left(S^{n+t}\right)$. Let $K^{\prime}$ be such a null-homotopy. Then $K=\Phi_{r-1}(f) \circ K^{\prime}$ is a null-homotopy of $\Phi_{r-1}(f) \circ \theta_{r-1}^{\prime}$. The set of all such difference elements $d(H, K)$ is called $\bar{\alpha} \cup_{r} \bar{\alpha}$.

TheOREM (3.1). Let $\bar{\alpha} \in G_{t}$ correspond to $\alpha \in E_{2}^{s, s+t}\left(S^{0}\right)$ and suppose that $r+1 \leqq \rho(t+r+1)$. Then there is an element of $\bar{\alpha} \cup_{r} \bar{\alpha}$ (as defined in Example A) which corresponds to $\alpha \cup_{r} \alpha \in E_{2}^{2 s-r, 2 s+2 t}\left(S^{0}\right)$.

ExAMPLe B. Let $t \equiv 2^{i+1}-1 \bmod 2^{i+1}$ and $r=2^{i}$ for $i=0,1,2$ or 3 . Then we may suppose that $\theta_{r-1}^{\prime}: S^{2 n+2 t+r-1} \rightarrow S^{2 n+2 t}$. Suppose that $\bar{\alpha}^{2}=0$. Let $K^{\prime}$ be a null-homotopy of $f \wedge f$ and let $K$ be the composite of $K^{\prime}$ with the cone on $\theta_{r-1}^{\prime}$. In this case the set of all such difference elements $d(H, K)$ is called $\bar{\alpha} \cup_{r} \bar{\alpha}$.

Theorem (3.2). Suppose that $\bar{\alpha} \in G_{t}$ corresponds to $\alpha \in E_{2}^{s, 8+t}\left(S^{0}\right)$ and that $t \equiv 2^{i+1}-1 \bmod 2^{i+1}, i=0,1,2$ or 3 . Suppose also that $\bar{\alpha}^{2}=0$ and that there is an honest relation $\delta_{m} \beta=\alpha^{2}$. Then $\bar{\alpha} \cup_{2^{i}} \bar{\alpha}$ (as defined in Example B) contains an element which corresponds to $\alpha \cup_{2}^{i} \alpha+h_{r} \beta$.

Notation. A formula $\delta^{s-r, t+1+s-r} \beta=\gamma \in E_{r}^{s, s+t}$ is called an honest relation if $\delta_{r+k+l}^{s-r-k, l+1+8-r-k}=0$ for $k \geqq 0, l \geqq 1$. A condition like this occurs in the work of Moss [7].

If $\gamma \in E_{2}^{s, s+z}$ and $\gamma^{\prime} \in E_{2}^{s^{\prime} s^{\prime}+k}$, then $\gamma+\gamma^{\prime}$ denotes $\gamma, \gamma+\gamma^{\prime}$ or $\gamma^{\prime}$ as $s<s^{\prime}, s=s^{\prime}$ or $s-s^{\prime}$, respectively.

\section{REFERENCES}

1. J. F. Adams, On the non-existence of elements of Hopf invariant one, Ann. of Math. (2) 72 (1960), 20-104.

2. - On the structure and applications of the Steenrod algebra, Comment. Math. Helv. 32 (1958), 180-214.

3. - - Vector fields on spheres, Ann. of Math. (2) 75 (1962), 603-632.

4. D. Husemoller, Fibre bundles, McGraw-Hill, New York, 1966.

5. D. S. Kahn, $A$ differential in the Adams spectral sequence, Proc. Amer. Math. Soc. 20 (1969), 188-190.

6. A. Liulevicius, The factorization of cyclic reduced powers by secondary cohomology operations, Mem. Amer. Math. Soc., No. 42, Amer. Math. Soc., Providence, R. I., 1962.

7. M. Moss, Secondary compositions and the Adams spectral sequence (to appear).

NORTHWESTERN UNIVERSITy, Evanston, IlLinois 60201 